\title{
Cytotoxic $T$ lymphocytes form an antigen-independent ring junction
}

\author{
Kristina Somersalo, ${ }^{1,2}$ Nadja Anikeeva, ${ }^{3,4}$ Tasha N. Sims, ${ }^{1,2}$ V. Kaye Thomas, ${ }^{1,2}$ \\ Roland K. Strong, 5 Thomas Spies, ${ }^{5}$ Tatiana Lebedeva, ${ }^{3,4}$ Yuri Sykulev, 3,4 \\ and Michael L. Dustin ${ }^{1,2}$
}

1Program in Molecular Pathogenesis, Skirball Institute of Biomolecular Medicine, and
${ }^{2}$ Department of Pathology, New York University School of Medicine, New York, New York, USA
${ }^{3}$ Department of Microbiology and Immunology, and
${ }^{4}$ Kimmel Cancer Institute, Thomas Jefferson University, Philadelphia, Pennsylvania, USA
${ }^{5}$ Division of Basic Sciences, Fred Hutchinson Cancer Research Center, Seattle, Washington, USA

Immunological synapses are organized cell-cell junctions between T lymphocytes and APCs composed of an adhesion ring, the peripheral supramolecular activation cluster (pSMAC), and a central $\mathrm{T}$ cell receptor cluster, the central supramolecular activation cluster (cSMAC). In CD8 ${ }^{+}$cytotoxic $\mathrm{T}$ lymphocytes, the immunological synapse is thought to facilitate specific killing by confining cytotoxic agents to the synaptic cleft. We have investigated the interaction of human CTLs and helper $\mathrm{T}$ cells with supported planar bilayers containing ICAM-1. This artificial substrate provides identical ligands to $\mathrm{CD}^{+}$and $\mathrm{CD}^{+} \mathrm{T}$ cells, allowing a quantitative comparison. We found that cytotoxic $\mathrm{T}$ lymphocytes form a ring junction similar to a PSMAC in response to high surface densities of ICAM-1 in the planar bilayer. MICA, a ligand for NKG2D, facilitated the ring junction formation at lower surface densities of ICAM-1. ICAM-1 and MICA are upregulated in tissues by inflammation- and stressassociated signaling, respectively. Activated $\mathrm{CD}^{+} \mathrm{T}$ cells formed fivefold more ring junctions than did activated $\mathrm{CD}^{+} \mathrm{T}$ cells. The ring junction contained lymphocyte function associated antigen-1 and talin, but did not trigger polarization and granule translocation to the interface. This result has specific implications for the mechanism of effective CTL hunting for antigen in tissues. Abnormalities in this process may alter CTL reactivity.

J. Clin. Invest. 113:49-57 (2004). doi:10.1172/JCI200419337.

\section{Introduction}

CTLs kill target cells bearing appropriate antigenic MHC-peptide (MHCp) complexes by forming an immunological synapse (IS) with target cells $(1,2)$. The CTL and target cell adhere using adhesion molecules including the integrin lymphocyte function associated antigen-1 (LFA-1, also known as CD11a/CD18) and its counter-receptor ICAM-1 (CD54) that form the adhesion ring junction or peripheral supramolecular activation complex (pSMAC) (2-4). Secretory granules containing perforin and granzymes appear to be directed to the CTL-target junction near the central T cell receptor (TCR) cluster or central supramolecular activation

Received for publication June 30, 2003, and accepted in revised form October 28, 2003.

Address correspondence to: Michael L. Dustin, Program in Molecular Pathogenesis, Skirball Institute of Biomolecular Medicine, 540 First Avenue, New York, New York 10016, USA. Phone: (212) 263-3207; Fax: (212) 263-5711;

E-mail: dustin@saturn.med.nyu.edu.

Conflict of interest: The authors have declared that no conflict of interest exists.

Nonstandard abbreviations used: MHC-peptide (MHCp); immunological synapse (IS); lymphocyte function associated antigen-1 (LFA-1); peripheral supramolecular activation cluster (pSMAC); T cell receptor (TCR); central supramolecular activation cluster (cSMAC); glycophosphatidylinositol (GPI); 6-histidine-tagged ( $\left.\mathrm{His}_{6}\right)$. complex (cSMAC) $(2,4)$. The dynamics of IS formation by CTLs have not been investigated. Defects in CTLmediated killing are associated with failure to destroy some immunogenic tumors and virally infected cells. Activated T cells hunting for antigenic MHCp complexes are attracted to peripheral tissues by chemoattractants and endothelial activation. Although adhesion is important for IS formation, the role of adhesion molecules in this hunting behavior, sometimes referred to as immune surveillance, is not known. Adhesion molecules like ICAM-1 are increased at sites of inflammation, and costimulatory ligands like MICA are upregulated on epithelial tumors as well as at sites of viral and bacterial infection and by inflammation $(5,6)$. These inflammation- and transformation-associated signals have the potential to facilitate productive CTL-target cell interactions by favoring more stable junction formation, but no data are available to address this issue.

Adhesive junction formation and IS formation can be quantified using supported planar bilayers. For example, helper T cell ISs can be fully reconstituted and visualized in real time when the APC is replaced by a supported planar bilayer containing only two molecules, ICAM-1 and an MHCp complex (7). We have taken this approach for analysis of dynamic early events in CTL IS formation and the antigen-independent interactions that may be relevant to the hunting process. We 
describe here the formation of an antigen-independent ring junction or PSMAC by human $\mathrm{CD} 8^{+} \mathrm{CTLs}$ with supported planar bilayers containing ICAM-1 and show that ring junction formation is increased by MICA. We propose that the ring junction is an adaptation of CTL to enhance the sensitivity of antigen recognition and the precision of target cell killing.

\section{Methods}

Cells. The human $\mathrm{CD}^{+} \mathrm{CTL}$ clone 68A62, which recognizes the ILKEPVHGV (IV9) peptide from HIV reverse transcriptase bound to HLA-A2 (8), was a gift from Bruce Walker (Harvard Medical School, Boston, Massachusetts, USA) (9). The human $\mathrm{CD}^{+}$CTL clone CER43, which recognizes the influenza matrix protein peptide GILGFVFTL (GL9) bound to HLA-A2 (10), was a gift from Antonio Lanzavecchia (Institute for Research in Biomedicine, Bellinzona, Switzerland) (11). After being stimulated with phytohemagglutinin or anti-CD3 $\mathrm{mAb}$, pooled irradiated human peripheral blood mononuclear cells, and IL-2, the CTLs were tested in 4-hour chromium-release assays (effector/target ratio, 5:1) with JY cells as target cells sensitized with various concentrations of cognate or irrelevant peptide. The peak period for CTL activity (that is, the lowest nonspecific lysis and highest sensitivity of cytolytic response) was approximately 2 weeks after stimulation, when proliferation had ceased. This time, however, varied with different restimulations and ranged from 1.5 to 3 weeks. The CTLs could be treated with LysoTracker Red DND-99 without a change in cytolytic activity.

Peripheral blood mononuclear cells were isolated from healthy donors. Samples were depleted of adherent cells by plastic adherence in complete medium (RPMI 1640 medium supplemented with $10 \%$ FCS, 10 mM HEPES, $2 \mathrm{mM}$ L-glutamine, $100 \mathrm{U} / \mathrm{ml}$ penicillin, $100 \mu \mathrm{g} / \mathrm{ml}$ streptomycin, and $50 \mu \mathrm{M} \beta$-mercaptoethanol) for 1 hour at $37^{\circ} \mathrm{C}$. Nonadherent cells were used for direct purification of CD69- $\mathrm{T}$ cells or were activated with antihuman CD3 (HB10166; American Type Culture Collection, Rockville, Maryland, USA) and anti-human CD28 (9.3) precoated on plastic at $10 \mu \mathrm{g} / \mathrm{ml}$ and $1 \mu \mathrm{g} / \mathrm{ml}$, respectively, and IL-2 at 50 units $/ \mathrm{ml}$ in the media. After 24 hours, the cells were transferred to a fresh flask and incubated at a density of $1 \times 10^{6}$ to $2 \times 10^{6}$ cells per milliliter for $6-8$ days with 50 units $/ \mathrm{ml}$ of IL-2. CD $8^{+}$and $\mathrm{CD}^{+} \mathrm{T}$ cells were isolated by negative selection using magnetic beads (StemCell Technologies, Vancouver, Canada) and $>90 \%$ purity was confirmed by flow cytometry. After stimulation, the cells were $>95 \% \mathrm{CD} 9^{+}$.

Antibodies and other chemicals. PA2.1 antibody (specific for polymorphic domain of HLA-A2) was kindly provided by Herman Eisen (Massachusetts Institute of Technology, Cambridge, Massachusetts, USA). Antihuman CD3 (UCHT-1) (mouse IgG1) and anti-human CD8 (UCHT-4) (mouse IgG2a) were from Diatec (Oslo, Norway); they were conjugated with Alexa Fluor 488 and Alexa Fluor 633 (Molecular Probes, Eugene, Oregon, USA), respectively. Anti-CD28 (9.3) was a gift from
John Hansen (University of Washington, Seattle, Washington, USA). Anti-Talin (clone 8d4) was from SigmaAldrich (St. Louis, Missouri, USA). Rabbit polyclonal $A b$ to mouse PKC- $\theta$ (SC-2121) was from Santa Cruz Biotechnology Inc. (Santa Cruz, California, USA). Alexa Fluor $488 \mathrm{~F}\left(\mathrm{ab}^{\prime}\right)_{2}$ and Alexa Fluor $660 \mathrm{~F}\left(\mathrm{ab}^{\prime}\right)_{2}$ fragments of goat anti-mouse IgG $(\mathrm{H}+\mathrm{L})$ were from Molecular Probes. Cy3, Cy5, and anti-rabbit IgG conjugated with Cy2 were purchased from Pharmacia-Amersham (Piscataway, New Jersey, USA).

Where indicated, T cells were labeled with LysoTracker Red DND-99 (Molecular Probes) at $60 \mathrm{nM}$ for $30 \mathrm{~min}-$ utes at $37^{\circ} \mathrm{C}$, with BODIPY NBD $\mathrm{C}_{6}$ ceramide at $5 \mu \mathrm{M}$ for 30 minutes at $4^{\circ} \mathrm{C}$, or with cholera toxin $\mathrm{B}$ subunit-Cy2 at a concentration of $2 \mu \mathrm{g} / \mathrm{ml}$ for 30 minutes at $4^{\circ} \mathrm{C}$. The cells were then washed three times with $20 \mathrm{mM}$ HEPES, $\mathrm{pH} 7.4,137 \mathrm{mM} \mathrm{NaCl}, 2 \mathrm{mM} \mathrm{Na}_{2} \mathrm{HPO}_{4}, 5 \mathrm{mM}$ D-glucose, $5 \mathrm{mM} \mathrm{KCl}$ and $1 \%$ human serum albumin (assay media) prior to being used in imaging experiments.

Planar bilayers. Human or mouse ICAM-1 with a glycophosphatidylinositol (GPI) anchor (ICAM-1-GPI) was purified from transfected BHK cells and labeled with Cy3 or Cy5 $(7,12)$. Cy5-ICAM-1-GPI was incorporated into liposomes, and planar bilayers were formed by incubation of liposomes with clean glass (7). The density of ICAM-1 in the bilayers was determined by immunoradiometric assay with iodinated RR $1 / 1$ for human ICAM-1 and YN1/1 for mouse ICAM-1. The density of ICAM- 1 was adjusted by mixing ICAM-1 containing liposomes with Egg lecithin liposomes at different ratios. 6-Histidine-tagged ( His $_{6}$ ) MHCp complexes were added to the bilayers by the inclusion of $40 \%$ DOGS-NTA (1,2-dioleoyl-sn-glycero-3-\{[N(5-amino-1carboxypentyl)iminodiacetic acid]succinyl\}) (Avanti Polar Lipids), which chelates $\mathrm{Ni}^{2+}$. The bilayers were blocked with $5 \%$ nonfat dried milk containing $100 \mu \mathrm{M}$ $\mathrm{NiCl}_{2}$. $\mathrm{His}_{6} \mathrm{HLA}-\mathrm{A} 2$ was expressed in insect cells, purified as described (13), and labeled with Alexa Fluor 488. HLA-A2 was loaded with peptide $(100 \mu \mathrm{M})$ overnight at $4^{\circ} \mathrm{C}$. HLA-A2 peptide $(100 \mathrm{nM})$ was incubated with the $\mathrm{Ni}^{+}$-chelating bilayer for 1 minute and washed with assay media. The flow cell was warmed up to $37^{\circ} \mathrm{C}$ and cells were injected in $100 \mu \mathrm{l}$ of assay media and imaged for 1-3 h on a wide-field or confocal fluorescence microscope. The density of HLA-A2 molecules on the bilayer was determined by binding of iodinated PA2.1. When $100 \mathrm{nM}$ of HLA-A2-peptide was incubated on bilayers containing $40 \mathrm{~mol} \%$ DOGS-NTA; a site density of $48 \pm 20$ molecules $/ \mu \mathrm{m}^{2}$ (No. of experiments $=3$ ) was attained. Soluble $\mathrm{His}_{6}$ MICA was prepared as previously described (14).

Immunofluorescence staining. Cells were fixed with warm $2 \%$ paraformaldehyde in PBS for 12 minutes, washed with $5 \mathrm{ml}$ of assay media, treated for 1 minute with $0.02-0.05 \%$ Triton X-100 and washed thoroughly with assay media. Then the cells were stained with fluorescent $\mathrm{mAb}$ or with primary and secondary antibodies.

Microscopy and image analysis. An inverted wide-field microscope with a cooled charge-coupled device camera (Yona Microscopes, Bethesda, Maryland, USA) or a Zeiss 


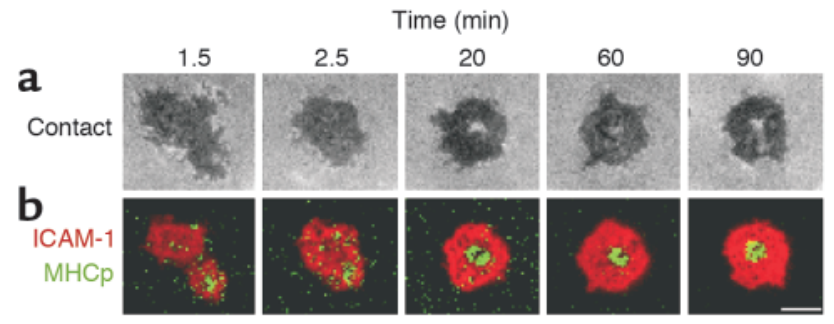

Figure 1

Kinetics of IS and contact formation by a CTL clone. A CER43 cell on a bilayer containing ICAM-1 and agonist MHCp complexes (GL9). (a) Interference reflection microscopy images showing contact area development (darker areas represent closer contact). (b) Fluorescence images show IS formation. Cy5-ICAM-1-GPI is red and Alexa Fluor 488-HLA-A2-GL9 is green. Scale bar: $5 \mu \mathrm{m}$.

LSM 510 confocal microscope (Zeiss, Thornwood, New York, USA) was used for multicolor fluorescence and interference reflection microscopy (15). Image processing and bilayer calibration was performed with IP-Lab software (Scanalytics, Vienna, Virginia, USA).

Statistical methods. Student's paired and unpaired $t$ tests were used. These data were normally distributed, as demonstrated by the $\chi^{2}$ test.

\section{Results}

Formation of mature immunological synapses by buman CTL clones. To study the dynamics of antigen-dependent CTL interactions, we used human CTL clones CER43 and 68A62. We used supported planar bilayers containing fluorescence-labeled HLA-A2-IV9 (CER43) or -GL9 (68A62) complexes at 40 molecules/ $\mu \mathrm{m}^{2}$ and ICAM- 1 at 200 molecules $/ \mu \mathrm{m}^{2}$. Although this system is less physiological than cellular targets, it provides better control over the molecules that are available, the images are more clearly resolved, and the images can be interpreted in terms of molecular interactions. The interaction of cell surface receptors TCR/CD8 and LFA-1 with their respective fluorescent ligands in the bilayer is visualized as fluorescence accumulation. CER 43 and $68 \mathrm{~A} 62$ cells initiated adhesion within 30 seconds of contact with the bilayer, and more than two thirds of cells were adherent within 8 minutes (Figure 1 and Table 1). IS formation was completed within $0.5-4$ minutes. The accumulation of MHCp complexes (green) was first observed in small areas surrounded by areas of ICAM-1 accumulation (Figure 1). These areas then coalesced into a single central cluster of MHCp complexes, the cSMAC, surrounded by a thick ring of ICAM- 1 accumulation, the PSMAC, together forming a mature IS (Figure 1).

CTLs form an antigen-independent ring junction. Surprisingly, IS-like structures were also formed in the absence of agonist MHCp complexes (Figure 2, a and b). We refer to these structures as ring junctions based on the presence of a distinct LFA-1-ICAM-1 ring but the absence of the central MHCp cluster. The first ring junctions were formed within 2 minutes of contact with the substrate. The ring junctions were transient, with an average duration of about 15 minutes (Table 1). T cells that were not forming ring junctions typically migrated over the surface, but were always stopped when ring junction patterns were observed. Roughly two thirds of either CTL clone would form at least one ring junction in a 1-hour observation period, and a single CTL was able to form one to four ring junctions within an hour (data not shown). Formation and dissolution of ring junctions reached a steady-state level of about $30 \%$ for each clone (Table 1). The density of LFA-1-ICAM-1 interactions in the IS and ring junction were not significantly different (Table 1). The number of LFA-1-ICAM-1 interactions per $\mathrm{PSMAC}$ was 6,000-7,000, representing about $10 \%$ of the total LFA- 1 molecules on the cell. The eccentricity, a measure of shape, was also not significantly different between the IS and the ring junction (Table 1). We were unable to detect any difference in the frequency or intensity of ring junction formation with ICAM-1 in the presence of irrelevant MHCp complexes or ICAM-1 alone (Figure 2c). Subsequent experiments focused on structures formed with only ICAM-1.

Molecular requirements for ring junction formation. To determine if ring junctions require CD8, we examined the CD8- CTL clone 115ix (N. Anikeeva and Y. Sykulev, unpublished observations). In three independent experiments, 115ix cells formed ring junctions with a 15-minute lag time (not shown). The steady-state level of ring junction formation was $18 \% \pm 6 \%$ with a duration of 2-8 minutes. Thus, CD8 is not required for the formation of ring junctions by CTL.

Because ICAM-1 expression is regulated by inflammation and can vary dramatically on cells from 0 to 1,000 molecules $/ \mu \mathrm{m}^{2}(5,16,17)$, we examined the relationship between ring junctions and ICAM- 1 density in the bilayers. Ring junctions were formed above an ICAM-1 density of 160 molecules $/ \mu \mathrm{m}^{2}$, while significant adhesion and ICAM-1 accumulation was observed at 40 molecules $/ \mathrm{\mu m}^{2}$ (Figure 2, $\mathrm{d}$ and e). Similar results were obtained for both CER43 and 68A62 CTL clones. Therefore, the threshold for efficient ring junction formation was much higher than the threshold for adhesion, suggesting that inflammation regulates ring junction formation through regulation of ICAM-1 expression.

Human $\mathrm{CD}^{+} \mathrm{T}$ cells express NKG2D, which mediates costimulation of CTLs (18). The ligands for NKG2D include MICA, the expression of which is upregulated by transformation and cellular stress (19). Therefore, we performed experiments to determine if MICA would modulate ring junction formation by CTL clones. $\mathrm{His}_{6}$ MICA was injected with the CTLs and attached to the bilayer through $\mathrm{Ni}^{2+}$-chelating lipid-His tag interactions. MICA significantly enhanced ring junction formation at low ICAM-1 densities (Figure 2d). This effect correlated with increased adhesion stimulated by MICA (Figure 2e). Therefore, the formation of ring junctions is responsive to at least two inflammatory/transformation-related signals, the ICAM-1 expression level and MICA.

Requirement for activation and comparison of $\mathrm{CD}^{+}$and $\mathrm{CD} 8^{+} \mathrm{T}$ cells. Freshly isolated CD69- human peripheral 
Immunological synapse and ring junction formation by $\mathrm{CD} 8^{+} \mathrm{CTL}$ clones

\begin{tabular}{|c|c|c|c|c|c|c|c|}
\hline & Without antigen & $N^{A}$ & $c^{B}$ & With antigen & $N$ & c & $P^{C}$ \\
\hline IS/presynapse (\% CER43): & $34 \pm 9$ & 10 & 744 & $76 \pm 15$ & 14 & 658 & $\leq 0.001$ \\
\hline IS/presynapse (\% 68A62) & $28 \pm 5$ & 4 & 271 & $71 \pm 6$ & 6 & 261 & $\leq 0.001$ \\
\hline IS/presynapse (\% CD8+ clones): & $32 \pm 10$ & 14 & 1015 & $75 \pm 16$ & 20 & 919 & $\leq 0.001$ \\
\hline pSMAC (min): & $18.9 \pm 18.1^{\mathrm{D}}$ & 3 & 64 & $1.6 \pm 1.3$ & 20 & 919 & $\leq 0.001$ \\
\hline $\operatorname{cSMAC}(\min ):$ & n.d. & & & $4.5 \pm 1.8$ & 11 & 237 & -- \\
\hline Duration (min): & $14 \pm 15$ & 10 & 744 & $>60$ & 14 & 658 & $\leq 0.001$ \\
\hline ICAM-1 molecules/ $\mu \mathrm{m}^{2}$ : & $167 \pm 32$ & 8 & 108 & $196 \pm 26$ & 5 & 101 & NS \\
\hline pSMAC area $\left(\mu \mathrm{m}^{2}\right):$ & $35 \pm 9$ & 8 & 108 & $37 \pm 10$ & 5 & 101 & NS \\
\hline cSMAC/central area $\left(\mu \mathrm{m}^{2}\right)$ : & $8 \pm 3$ & 8 & 108 & $12 \pm 4$ & 5 & 101 & NS \\
\hline Eccentricity ${ }^{\mathrm{E}}$ of ring: & $0.6 \pm 0.1$ & 8 & 108 & $0.5 \pm 0.1$ & 5 & 101 & NS \\
\hline Eccentricity ${ }^{\mathrm{E}}$ of central zone: & $0.7 \pm 0.0$ & 8 & 108 & $0.6 \pm 0.1$ & 5 & 101 & NS \\
\hline Migration morphology (\%): & $72 \pm 15$ & 10 & 744 & $0 \pm 0$ & 14 & 658 & $\leq 0.001$ \\
\hline CD3 accumulation (\%) & $25 \pm 5$ & 5 & 113 & $88 \pm 6$ & 5 & 258 & $\leq 0.001$ \\
\hline Area of CD3 accumulation $\left(\mu \mathrm{m}^{2}\right)$ & $3.9 \pm 5.0$ & 5 & 113 & $10.9 \pm 5.9$ & 5 & 258 & $\leq 0.01$ \\
\hline CD3 molecules ${ }^{F}$ in ${ }^{2 S M A C}$ & $1 \pm 1$ & 5 & 113 & $5 \pm 2$ & 5 & 258 & $\leq 0.01$ \\
\hline CD8 accumulation (\%) & $30 \pm 3$ & 4 & 95 & $87 \pm 6$ & 4 & 219 & $\leq 0.001$ \\
\hline Area of CD8 accumulation $\left(\mu \mathrm{m}^{2}\right)$ & $4.1 \pm 5.1$ & 4 & 95 & $7.5 \pm 4.8$ & 4 & 219 & NS \\
\hline CD8 molecules ${ }^{F}$ in $c S M A C$ & $1 \pm 1$ & 4 & 95 & $3 \pm 1$ & 4 & 219 & $\leq 0.05$ \\
\hline Cholera toxin Cy2 in center (\%) & $30 \pm 6$ & 2 & 23 & $100 \pm 0$ & 2 & 53 & $\leq 0.001$ \\
\hline Golgi complex polarization ${ }^{G}$ & $3 \pm 1$ & 3 & 63 & $90 \pm 7$ & 3 & 87 & $\leq 0.001$ \\
\hline Granule polarization ${ }^{G}$ & $2 \pm 0$ & 3 & 63 & $89 \pm 6$ & 3 & 87 & $\leq 0.001$ \\
\hline Talin & $100 \pm 0$ & 4 & 122 & $100 \pm 0$ & 4 & 130 & NS \\
\hline PKC- $\theta$ in $\mathrm{CSMAC}$ & $0 \pm 0$ & 3 & 42 & $23 \pm 2$ & 3 & 49 & $\leq 0.001$ \\
\hline pSMAC & $0 \pm 0$ & 3 & 42 & $67 \pm 19$ & 3 & 49 & $\leq 0.001$ \\
\hline
\end{tabular}

${ }^{A} N=$ number of experiments. ${ }^{B} \mathrm{C}=$ total number of cells analyzed. ${ }^{C} P=$ probability that the difference is due to random chance $\left(t\right.$ test). ${ }^{D} C e l l s$ on bilayer with out antigen form many PSMACs in series. The time required for formation of the first pSMAC after initial adhesion is indicated. EEccentricity is a measure of the circularity of the ellipse. It is 0 for perfect circles and approaches 1 for extreme ellipses. ${ }^{F}$ CD 3 and CD8 molecules in cSMACs are expressed as relative density. TThe percentage of cells in which the Golgi complex or granules are less than $1 \mu \mathrm{m}$ from bilayer. To assign a score of "polarized" we required more than half of the Golgi/granules to be $1 \mu \mathrm{m}$ from the surface. n.d., not done.

blood $\mathrm{CD} 8^{+} \mathrm{T}$ cells lacked cytolytic granules and were unable to form ring junctions, but also adhered poorly (Table 2). CXCL12 chemokine increased adhesion of fresh cells, but this did not induce ring junction formation. Different types of effector cells were directly compared by isolation of $\mathrm{CD} 4^{+}$and $\mathrm{CD} 8^{+}$effector cells from cultures of peripheral blood $\mathrm{T}$ cells stimulated with anti$\mathrm{CD} 3$ and anti-CD28. CD8 ${ }^{+}$effector $\mathrm{T}$ cells formed more than fivefold more ring junctions than did $\mathrm{CD}^{+}$effector $\mathrm{T}$ cells isolated from the same cocultures (Figure 3 ). Since the $\mathrm{CD} 4^{+} \mathrm{T}$ cell population was slightly contaminated by $\mathrm{CD}^{+} \mathrm{T}$ cells $(\leq 5 \%)$, it is possible that the ring junctions in the $\mathrm{CD}^{+}$fraction were formed by contaminating $\mathrm{CD}^{+} \mathrm{T}$ cells. To determine if effector cells derived from primary culture of naive $\mathrm{T}$ cells could form ring junctions, we tested effector $\mathrm{T}$ cells derived from human umbilical cord blood samples, which are highly enriched in naive $T$ cells, that had been stimulated on anti-CD3 and anti-CD28. CD8 ${ }^{+}$effector $\mathrm{T}$ cells from cord blood formed threefold fewer rings than did $\mathrm{CD}^{+}$effector cells from parallel cultures of adult cells (Table 2).

Location of $C D 3$ and $C D 8$ and lipid raft in the ring junction and IS. The central hole in the ring junction might be a site of sensitive antigen recognition if TCRs and the coreceptor CD8 were present. CD3 and CD8 localization were determined by immunofluorescence on cells fixed during interaction with the bilayers. CD3 and
CD8 accumulated in nearly $90 \%$ of cell contacts in the presence of agonist MHCp complexes (Figure 4, a and c, and Table 1). In many ISs, a central hole was detected in the CD8 pattern (Figure 4, b and c). CD3 and CD8 were present in the central region of the ring junction on ICAM- 1 alone, but they were only enriched in $30 \%$ of contacts, and the degree of enrichment was lower than in the IS (Figure 4, j, 1, and $\mathrm{m}$, and Table 1). CD3 was also weakly accumulated in the centers of ring junctions formed by the CD8- CTL line 115ix (Figure 4k).

Signaling proteins associated with "lipid rafts" in T cells are important for kinase cascades, so we determined if these are located in the IS and ring junction by staining of fixed cells with cholera toxin B subunit in fixed cells. Cholera toxin B subunit binding sites were enriched in the center of the CTL IS (Table 1) (20). Accumulation of cholera toxin binding sites in the ring junction was similar to the level of CD3 and CD8 accumulation, with a third of synapses showing a clear accumulation in the central region (Table 1).

Location of talin and PKC- $\theta$. Talin is implicated in integrin regulation and is a classical marker for the pSMAC of the IS $(21,22)$. Therefore, we sought to determine if talin is localized to the ring junction by immunofluorescence of fixed cells. Consistent with results for murine CTLs (2), talin showed considerable accumulation in the PSMAC formed by human 

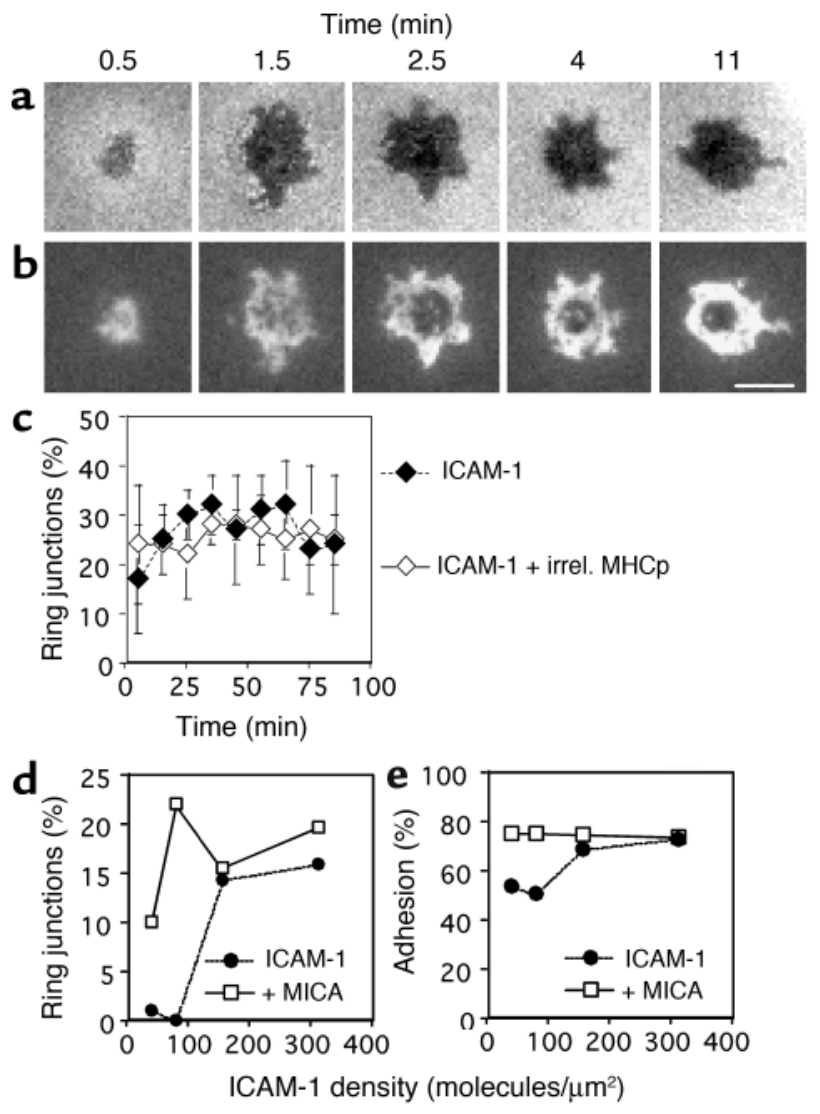

CTLs and was absent from the cSMAC (Figure 4, d and e). Ring junctions also accumulated talin in the pSMAC to the same extent as in the IS (Figure 4, $n$ and $\mathrm{o}$, and Table 1). Talin also accumulated in migratory contacts in a manner that mirrored the ICAM-1 accumulation (Figure 4, p and q). This is consistent with a recent report that talin binding to integrin cytoplasmic domains is a major common pathway for integrin activation $(23,24)$.

PKC $-\theta$ translocates to the cSMAC during murine $\mathrm{CD}^{+}$and $\mathrm{CD}^{+}{ }^{+} \mathrm{T}$ cell activation $(3,22)$. Therefore, we also investigated PKC- $\theta$ localization in the ring junctions and ISs formed by human CTLs by immunofluorescence after fixation. As expected, PKC- $\theta$ stained the cSMAC in a significant proportion of ISs but a greater proportion (four fifths) of ISs has PKC- $\theta$ accumulated in subregions of the pSMAC (Table 1 and Figure 4, h and i). The clusters of PKC- $\theta$ staining in the PSMAC of the IS appeared to be in small spots where ICAM- 1 was excluded. In contrast, PKC- $\theta$ did not associate with the ring junction (Table 1 and Figure 4, $r$ and s).

Location of granules and Golgi apparatus. A major function of the CTL synapse is directed secretion (2). We monitored the cytolytic granules and Golgi apparatuses of live CTLs forming ISs or ring junctions $(2,25)$. In ISs, most of the granules and the Golgi were concentrated within $1 \mu \mathrm{m}$ of the center of the IS (Figure 5 and Table 1). In contrast, granules were randomly distributed and the Golgi was typically located away from the ring junction (Figure 5 and Table 1).

\section{Figure 2}

The kinetics of and the irrelevant MHCp complex and ICAM-1 density dependence of ring junction and contact formation by CTL clones. (a and b) A CER43 cell on a bilayer containing 200 molecules $/ \mu \mathrm{m}^{2}$ Cy3-ICAM-1-GPI alone. (a) Interference reflection microscopy images showing contact development (darker areas represent closer contact). (b) Fluorescence images showing LFA-1-ICAM-1 interaction during ring junction development. Cy3-ICAM-1 in grayscale (white represents the highest ICAM-1 density). The mean duration of transient ring junctions is 15 minutes, and a cell can make one to four ring junctions within an hour, with intervening migration. Scale bar: $5 \mu \mathrm{m}$. (c) Effect of irrelevant (irrel.) MHCp complexes on ring junction formation. The kinetics of ring junction formation were assessed for populations of CER43 cells on 200 molecules/ $\mu \mathrm{m}^{2}$ Cy3-ICAM-1GPI (filled diamonds) or 40 molecules $/ \mu \mathrm{m}^{2}$ irrelevant Alexa 488 -HLAA2-IV9 (open diamonds). Ring junction formation reached a steady state within 15 minutes. No significant difference was detected without or with irrelevant MHCp complexes. (d) Percentage of ring junction formation by CTL clone 68A62 as a function of ICAM- 1 density in the absence (filled circles) and presence (open squares) of $100 \mathrm{nM}$ MICA on bilayers containing $50 \% \mathrm{Ni}^{2+} /$ NTA lipids. (e) Percentage of adhering 68A62 CTL clone cells as a function of ICAM-1 density in the absence (filled circles) and presence (open squares) of $100 \mathrm{nM}$ MICA on bilayers containing $50 \% \mathrm{Ni}^{2+} / \mathrm{NTA}$ lipids. Data in $\mathbf{d}$ and $\mathbf{e}$ are representative of data for 68A62 and CER43 CTL clones. In each case, over 1,000 cell contacts were analyzed to generate the graphs.

\section{Discussion}

In this study we evaluated the dynamics of human CTL IS formation and examined antigen-independent interactions of CTLs with supported planar bilayers. First, we demonstrated that ICAM-1 and agonist MHCP complexes in a mobile lipid membrane are sufficient to form a stable CTL IS. Second, we found that human CTL clones and primary activated human $\mathrm{CD} 8^{+} \mathrm{T}$ cells from adult peripheral blood form antigen-independent ring junctions with the planar bilayer. $\mathrm{CD} 8^{+}$effector cells from adults were at least fivefold more effective than $\mathrm{CD}^{+}$ effector cells from adults and were threefold more effective than $\mathrm{CD}^{+}$effector cells from cord blood at forming ring junctions. We cannot conclude that $\mathrm{CD} 4^{+} \mathrm{T}$ cells form ring junctions because of the presence of $5 \%$ or less contaminating $\mathrm{CD}^{+}$cells in the $\mathrm{CD} 4^{+}$fraction. Third, the formation of ring junctions was dependent upon high ICAM-1 density, but could be induced at lower ICAM-1 density in the presence of MICA, a ligand for the costimulatory NKG2D receptor on the CD8 $\mathrm{T}$ cells. Fourth, the antigen-independent ring junction did not polarize the secretory apparatus.

\section{Table 2}

Ring junction formation by other cell types

$\begin{array}{lcccc}\text { Peripheral cells } & & & \\ \text { CD8 }{ }^{+} \text {basal } & 17.5 & 2 & 2 & 328 \\ \text { CD8 }{ }^{+} \text {anti-CD3 effectors } & 72.5 & 18.5 & 2 & 261 \\ \text { Cord blood } & 3 & 0 & 1 & 212 \\ \text { CD8 }{ }^{+} \text {basal } & 39.5 & 6.5 & 2 & 208 \\ \text { CD8 }{ }^{+} \text {anti-CD3 effectors } & 39.5 & \end{array}$

AThe percentage of input cells forming presynapses. 


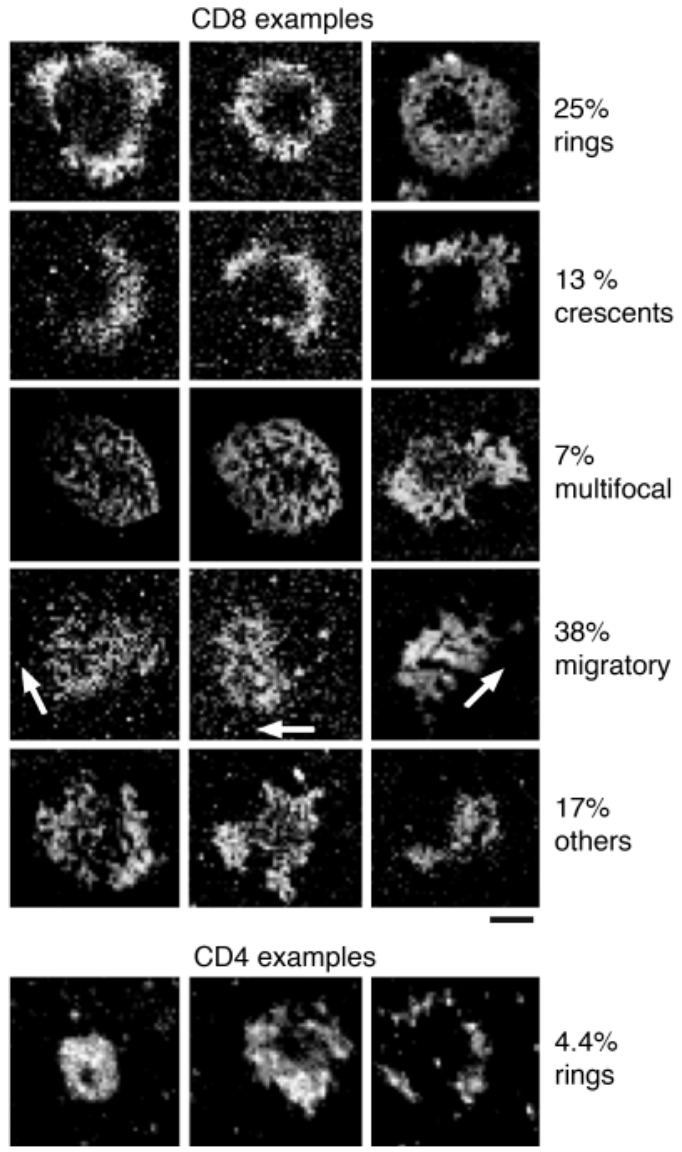

The experiments with the CTL IS provided several surprises. No nascent immunological synapse with TCR engagement in the periphery was detected. This contrasts with IS formation by CD4 T cells, in which the TCR and MHCp complex initially interact in peripheral microclusters $(7,26)$. We propose below that this is an important adaptation for CTL function. The finding that CD8 was localized in the CSMAC was consistent with studies of CD4, but the finding that many cSMACs had a central hole lacking $\mathrm{CD} 8$, but containing $\mathrm{CD} 3$, was surprising. In CD4 $\mathrm{T}$ cells there is also exclusion of coreceptors from the cSMAC to the PSMAC, but in CD8 T cells this segregation may take place within the cSMAC itself. Another unexpected finding was that PKC- $\theta$, which is only reported to localize in the $\operatorname{cSMAC}(3)$, was typically found to be scattered in the pSMAC in the CTL IS. Its not clear if this is another adaptation of CTLs, which may use the central region as a secretory domain (2), such that some signaling machinery is relocated to the periphery.

We demonstrated ring junction formation in response to ICAM- 1 and its augmented through a distinct antigenindependent signal, MICA. ICAM- 1 is one of several ligands for the leukocyte integrin LFA-1, but stands out as being the most potent ligand in cell-adhesion assays (27). ICAM- 1 is an inducible adhesion molecule and this may be relevant to its role in the formation of ring junctions (5). ICAM-1 is constitutively expressed at low levels on endothelial cells, some epithelial cells, and myeloid cells

\section{Figure 3}

Gallery of antigen-independent contacts formed by activated human peripheral blood $\mathrm{CD} 8^{+} \mathrm{T}$ cells. Anti-CD3/CD28-stimulated peripheral blood $\mathrm{CD}^{+}$and $\mathrm{CD}^{+} \mathrm{T}$ cell contacts on Cy3-ICAM-1-GPI in the bilayer (the highest ICAM-1 density is white). The patterns were categorized based on the presence and completeness of PSMAC morphology. Only ring junction examples are provided for $\mathrm{CD} 4^{+} \mathrm{T}$ cells. The percentage of cells in each category is along the right margin. Results are representative of three experiments. Scale bar: $5 \mu \mathrm{m}$.

$(16,17)$. Activation of lymphocytes and myeloid cells leads to induction or increases in ICAM-1 expression (5). ICAM-1 expression on epithelial cells and astrocytes is increased by treatment with interferon- $\gamma(5,17,28)$. ICAM- 1 expression on fibroblasts and endothelial cells is increased by interferon- $\gamma$, tumor necrosis factor, interleukin-1, and lipopolysaccharide $(5,17)$. The densities of ICAM-1 that trigger ring junction formation are consistent with levels of ICAM-1 expressed on activated lymphoid and myeloid cells and cytokine-activated nonhematopoietic cells. While ICAM-1 expression alone is not thought to trigger activation of immune or inflammatory responses (29), it is likely that increasing ICAM-1 expression facilitates the effectiveness of $\mathrm{T}$ cells' hunting for targets. Consistent with this, ICAM-1 is downregulated by the Kaposi sarcoma K5 protein, contributing to defects in T cell recognition (30). Thus, increased ICAM1 expression in response to inflammation in tissues may augment ring junction formation by CTLs patrolling the tissue to heighten tissue surveillance. MICA is an activating ligand for NKG2D on natural killer cells and a costimulatory ligand on $\mathrm{CD}^{+} \mathrm{T}$ cells $(18,31)$. We found that MICA greatly enhanced ring junction formation at low densities of ICAM-1. The conditions under which ICAM-1 and MICA are expressed are only partially overlapping, such that either strong upregulation of ICAM-1 by inflammatory cytokines or upregulation of MICA by transformation or cellular stress at low levels of ICAM-1 can lead to the formation of ring junctions. When high levels of ICAM-1 were combined with MICA, ring junction formation was not increased further.

Recently, Trautmann and colleagues described an antigen-independent IS between naive murine $T$ cells and dendritic cells (32). This structure shows PKC- $\theta$ recruitment and microtubule-organizing center polarization and does not fully activate the naive $\mathrm{T}$ cells, but may provide survival signals. Like the ring junction, $\mathrm{MHC}$ molecules are not required for the antigen-independent ISs of naive $T$ cells with dendritic cells. We do not refer to the ring junctions reported here as synapses, because they lack polarity in their secretory machinery and lack recruitment of PKC- $\theta$. The signals that lead to antigen-independent IS formation by naive $T$ cells and dendritic cells are unknown, but may include other combinations of adhesion and costimulatory molecules provided by the dendritic cell, which are absent in our planar bilayer model.

The antigen-independent ring junction may be an adaptation for CTL function. The antigen-dependent PSMAC formation by CTLs was rapid and appeared to 

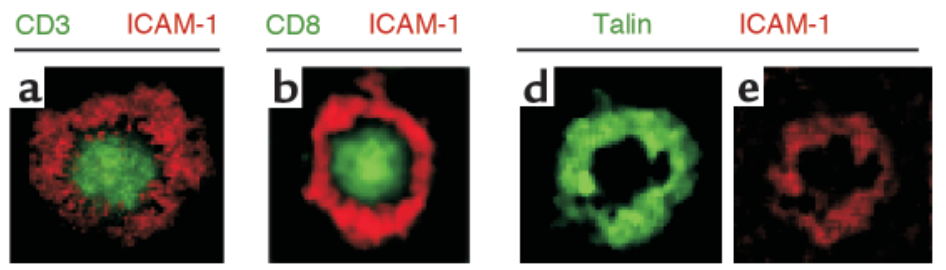

ICAM-1

IS
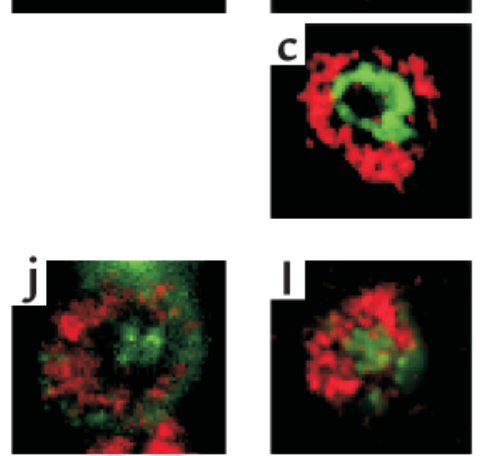

Ring

junctions

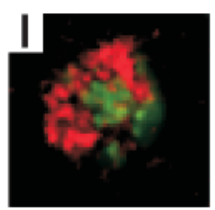

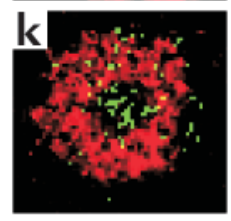
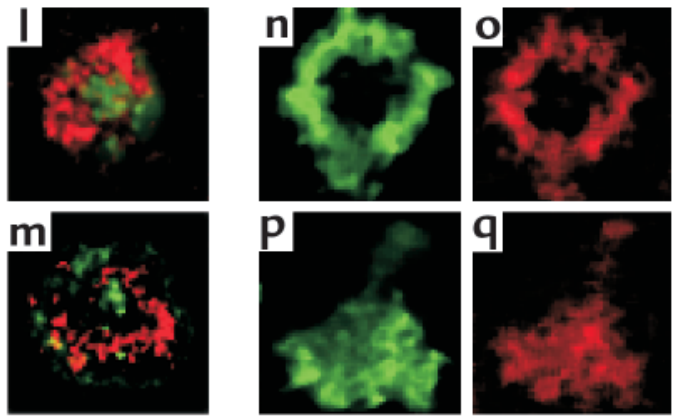
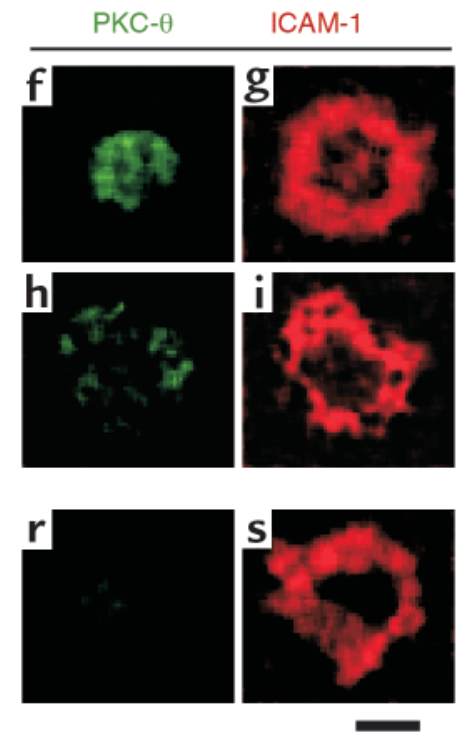

Figure 4

Confocal images of TCR, CD8, talin, and PKC- $\theta$ distribution of CTL clones. CTL clone CER43 was layered on bilayer containing 200 molecules/ $\mu \mathrm{m}^{2}$ Cy3-ICAM-1-GPI with or without unstained agonist MHCp complexes (a-i) or containing 200 molecules/ $\mu m^{2}$ Cy3-ICAM-1-GPI only (j, I-s). In k, the CD8-deficient CTL clone 115ix was layered on a bilayer containing 200 molecules $/ \mu \mathrm{m}^{2} \mathrm{Cy} 3-\mathrm{ICAM}-1-\mathrm{GPI}$ only. After 30 minutes of incubation, cells were fixed, permeabilized, and stained with the indicated antibodies. Fluorescence was imaged with a laser-scanning confocal microscope. The green signal in $\mathbf{j}$ and $\mathbf{k}$ is increased to reveal the presence of levels of CD3 staining above the baseline density. (k) CD3 can accumulate in the center of the ring junction without CD8. (b and $\mathbf{c}$ ) Examples of CD8 accumulations (green) that are filled (b) or have central holes (c). (I and $\mathbf{m}$ ) Examples of different levels of CD8 in ring junctions, which are not as enriched as in IS. Pairs $\mathbf{d} / \mathbf{e}, \mathbf{n} / \mathbf{o}$, and $\mathbf{p} / \mathbf{q}$ are the same contacts with talin (green) or ICAM-1 (red) fluorescence; $\mathbf{n} / \mathbf{o}$ is a ring junction, while $\mathbf{p} / \mathbf{q}$ is a motile junction, which also shows talin redistribution. Pairs $\mathbf{f} / \mathbf{g}$, $\mathbf{h} / \mathbf{i}$, and $\mathbf{r} / \mathbf{s}$ are the same contacts stained for PKC $-\theta$ (green) and ICAM- 1 (red); $\mathbf{f} / \mathbf{g}$ shows the classical central localization of PKC- $\theta$ compared with $\mathbf{h} / \mathbf{i}$, which shows the more frequent localization of PKC- $\theta$ to the microaggregates in the PSMAC; $\mathbf{r} / \mathbf{s}$ shows that PKC- $\theta$ was not detected in the ring junctions. The images were processed with a median filter (radius 4 pixels [pixel $=.2 \mu \mathrm{m}$ ]). Scale bar: $5 \mu \mathrm{m}$.

precede detectable MHCp complex accumulation, unlike the situation with helper T cells, in which initial MHCp complex accumulation occurs in the periphery of the contact area before a ring junction forms. We propose that the ring junction is one manifestation of a general difference in the CTL cytoskeleton, compared with helper T cells, which predisposes CTL to rapid ring junction formation in response LFA-1 ligation above a threshold level. This change may be fully enacted through restimulation or in the presence of cytokines from activated memory cells, since effector cells derived from cord blood were less effective at forming ring junctions. This threshold can be altered by signals like MICA. The ring junction causes the CTL to pause and form a central domain with $\mathrm{CD} 3, \mathrm{CD} 8$, and lipid rafts, an ideal signal reception surface, but does not mobilize the secretory apparatus. We would suggest that the cell is on "high alert" but its weapons are not "armed" yet. The ring junction of CTLs was observed on a very simple substrate, a planar bilayer containing only one adhesion molecule, strongly suggesting that ring junctions reflect an unanticipated response to signals through the integrin LFA- 1 in CTLs. It is possible that TCR interac- tions with self MHCp complexes, which we could not evaluate here, may contribute to this process in physiological cell-cell interactions.

Lymphoid and myeloid cells form two different types of ring junctions. One type comprises podosomes which are ring junctions that form on a much smaller scale, each about $1 \mu \mathrm{m}$ across, within contacts (33). Despite this difference in size, they have a composition similar to that of the ring junctions that we have reported here. The second set are larger-scale ring junctions formed by the interaction of myeloid cells and osteoclasts with planar substrates (34). Ring junctions can be triggered by the interaction of immune complexes with Fc receptors and thus may be directly analogous to a mature IS (35). Polymorphonuclear phagocytes form protected adhesion zones, which exclude antibodies, on fibrinogen, a ligand for integrins CD11b and $\mathrm{CD} 11 \mathrm{c}(36)$. Based on these results, ring junctions are thought to form "gasket-like" barriers to establish regulated extracellular compartments for cell communication, effector functions, or matrix degradation.

CTL ring junctions were transient. CTLs could form up to four ring junctions per hour. This tran- 

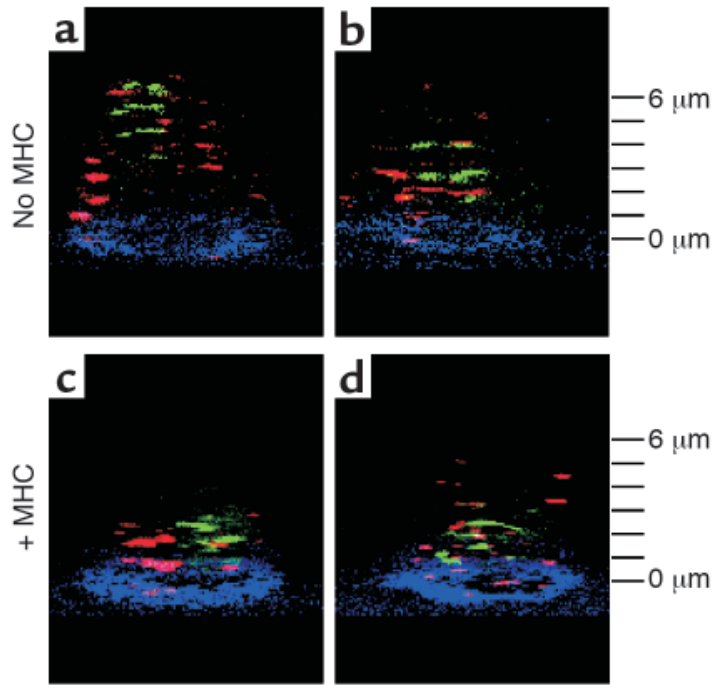

ICAM-1

Granules

sient nature may make the ring junctions physically weaker than antigen-dependent CTL pSMACs, despite their having similar numbers of LFA1-ICAM-1 interactions. It is also possible that the molecular interactions in the cSMAC of the IS may contribute a significant stabilizing influence. This could be evaluated in future studies by applying calibrated shear forces or by micromanipulation (37, 38). The timing mechanism involved in the cyclical nature of ring junction formation is not known. However, the frequency of ring junction formation is strikingly similar to the classical frequency of serial target cell killing by CTLs $(39,40)$.

Our data suggest that CTLs are predisposed to form ring junctions in response to high densities of ICAM-1 in combination with other antigen-independent signals like MICA. We envision two functions for CTL ring junctions. Rapid formation or preformation of a ring junction may ensure that release of cytotoxic granules takes place only within an area surrounded by the adhesive gasket. Another function of the antigen-independent ring junction may be to intensify

\section{Figure 6}

Model for IS formation by helper T cells and CTLs. Based on prior reports, the helper $T$ cells migrate on substrates with ICAM-1 (dark gray) alone. When agonist MHCp complexes are encountered, the cells form a nascent IS with ICAM-1 in the center and MHCp complexes (light gray) on the periphery. This pattern then inverts to form the mature IS with ICAM- 1 in a ring and MHCP complexes in the center. Based on this study, we propose that CTLs on ICAM-1 alone alternate about four times per hour between ring junctions in which ICAM- 1 is organized in a ring and typical migratory morphology. We propose that the CTL ring junction constitutes a functional "presynapse" that intensifies immune surveillance. When antigen is encountered, CTLs form an IS without going through distinct nascent and mature stages. We propose that this "short cut" saves the CTL time and ensures that an adhesive "gasket" is formed before cytolytic substances are released into the synaptic cleft.

\section{Figure 5}

Three-dimensional distribution of granules and Golgi complexes in CTLs forming ISs or ring junctions. Tilted three-dimensional views of CTL CER43 cells stained with LysoTracker red (granules; red) and BODIPY ceramide (Golgi; green). Cells were layered on bilayer containing human Cy5-ICAM-1-GPI (blue) only (a and $\mathbf{b}$ ), where they form a ring junction, or on a bilayer containing human Cy5-ICAM-1-GPI (blue) and unstained agonist MHCp complexes ( $\mathbf{c}$ and $\mathbf{d}$ ), where they form an IS. Two examples are provided for each substrate to illustrate the range of patterns that were typical of many more cells scored and summarized in Table 1. All images were acquired within an hour of contact with the bilayer. The $z$-stack interval is $1 \mu \mathrm{m}$ with the bilayer level defined at zero.

immune surveillance by increasing the chances of finding rare MHCp complexes. The cost of ring junction formation is that the $\mathrm{T}$ cell temporarily stops its hunting, which might reduce the number of cells it can scan, but the benefit may be in a reduction of missed targets with low amounts of MHCp complexes or those MHCp complexes that weakly bound to TCR. The effective clearance of a virus or eradication of tumor may depend heavily upon its not missing rare MHCp complexes. Our data fit with a general model for two pathways to IS formation (Figure 6). One pathway is antigen independent through a ring junction (CTLs in inflamed sites) and one pathway is antigen dependent through a nascent IS (helper $\mathrm{T}$ cells). Recently, a computational model also suggested fundamental differences between helper $T$ cell and CTL IS formation that are consistent with our data (41). The ring junction of the CTL may constitute a specific functional structure that could be described as a "presynapse." We propose that the ability to form this presynapse sets the stage for a sensitive and selective CTL killing of targets (42).

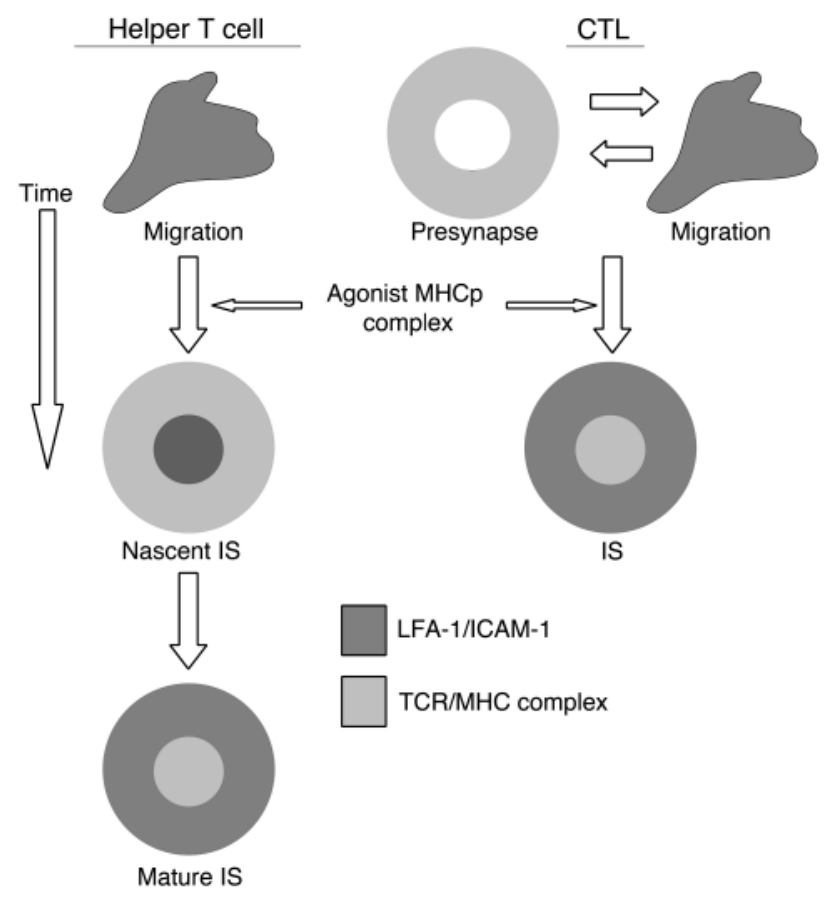




\section{Acknowledgments}

We thank A. Diefenbach and A. Shaw for discussions on NKG2D. We thank N. Desai and R. Houdei for preparation of the ICAM-1-GPI liposomes. We thank R. Rothlein (Boehringer Ingelheim, Ridgefield, Connecticut, USA), B.D. Walker (Harvard Medical School, Boston, Massachusetts, USA), S. Kalams (Vanderbilt University Medical Center, Nashville, Tennessee, USA), A. Lanzavecchia (Institute for Research in Biomedicine, Bellinzona, Switzerland), and $\mathrm{H}$. Eisen (Massachusetts Institute of Technology, Boston, Massachusetts, USA) for reagents and CTL clones. We thank members of the Dustin and Sykulev labs for support and advice. This work was supported by NIH research grants AI3254 and AI5282 to Y. Sykulev, AI44931 and AI5282 to M.L. Dustin, AI30581 to T. Spies, and AI48675 to R.K. Strong. N. Anikeeva Y. Sykulev and T. Lebedeva were supported in part by National Research Service Awards Training Program for AIDS Research 5-T32-AI07523, in Cancer Immunology and in Molecular Oncology and Immunology 5-T32-CA09683, respectively. K. Somersalo was supported in part by the Finnish Academy of Sciences. T.N. Sims is supported by a seed grant from the National Psoriasis Foundation/USA. M.L. Dustin is supported by the Irene Diamond Fund.

1. Norcross, M.A. 1984. A synaptic basis for T-lymphocyte activation. Ann. Immunol. (Paris). 135D:113-134.

2.Stinchcombe, J.C., Bossi, G., Booth, S., and Griffiths, G.M. 2001. The immunological synapse of CTL contains a secretory domain and membrane bridges. Immunity. 15:751-761.

3. Potter, T.A., Grebe, K., Freiberg, B., and Kupfer, A. 2001. Formation of supramolecular activation clusters on fresh ex vivo CD8+ T cells after engagement of the T cell antigen receptor and CD8 by antigen-presenting cells. Proc. Natl. Acad. Sci. U. S. A. 98:12624-12629.

4. Kuhn, J.R., and Poenie, M. 2002. Dynamic polarization of the microtubule cytoskeleton during CTL-mediated killing. Immunity. 16:111-121.

5. Dustin, M.L., Rothlein, R., Bhan, A.K., Dinarello, C.A., and Springer, T.A. 1986. Induction by IL-1 and interferon, tissue distribution, biochemistry, and function of a natural adherence molecule (ICAM-1). J. Immunol. 137:245-254.

6. Bauer, S., et al. 1999. Activation of NK cells and T cells by NKG2D, a receptor for stress-inducible MICA. Science. 285:727-729.

7. Grakoui, A., et al. 1999. The immunological synapse: A molecular machine controlling T cell activation. Science. 285:221-227.

8. Tsomides, T.J., Walker, B.D., and Eisen, H.N. 1991. An optimal viral peptide recognized by $\mathrm{CD}^{+} \mathrm{T}$ cells binds very tightly to the restricting class I major histocompatibility complex protein on intact cells but not to the purified class I protein. Proc. Natl. Acad. Sci. U. S. A. 88:11276-11280.

9. Walker, B.D., et al. 1989. Long-term culture and fine specificity of human cytotoxic T-lymphocyte clones reactive with human immunodeficiency virus type 1. Proc. Natl. Acad. Sci. U. S. A. 86:9514-9518.

10. Gotch, F., Rothbard, J., Howland, K., Townsend, A., and McMichael, A. 1987. Cytotoxic T lymphocytes recognize a fragment of influenza virus matrix protein in association with HLA-A2. Nature. 326:881-882.

11. Valitutti, S., Muller, S., Dessing, M., and Lanzavecchia, A. 1996. Different responses are elicited in cytotoxic $\mathrm{T}$ lymphocytes by different levels of T cell receptor occupancy. J. Exp. Med. 183:1917-1921.

12. Dustin, M.L., et al. 1998. A novel adapter protein orchestrates receptor patterning and cytoskeletal polarity in T cell contacts. Cell. 94:667-677.

13. Anikeeva, N., et al. 2003. Distinct molecular mechanisms account for the specificity of two different T-cell receptors. Biochemistry. 42:4709-4716.

14. Steinle, A., et al. 2001. Interactions of human NKG2D with its ligands MICA, MICB, and homologs of the mouse RAE-1 protein family. Immunogenetics. 53:279-287.

15. Izzard, C.S., and Lochner, L. 1976. Cell-to-cell substrate contacts in living fibroblasts: an interference reflection study with an evaluation of the technique. J. Cell Sci. 21:129-159.

16. Dustin, M.L., Singer, K.H., Tuck, D.T., and Springer, T.A. 1988. Adhe- sion of T lymphoblasts to epidermal keratinocytes is regulated by interferon gamma and is mediated by intercellular adhesion molecule- 1 (ICAM-1). J. Exp. Med. 167:1323-1340.

17. Dustin, M.L., and Springer, T.A. 1988. Lymphocyte function associated antigen-1 (LFA-1) interaction with intercellular adhesion molecule-1 (ICAM-1) is one of at least three mechanisms for lymphocyte adhesion to cultured endothelial cells. J. Cell Biol. 107:321-331.

18. Groh, V., et al. 2001. Costimulation of CD $8 \alpha \beta$ T cells by NKG2D via engagement by MIC induced on virus-infected cells. Nat. Immunol. 2:255-260

19. Molinero, L.L., Gruber, M., Leoni, J., Woscoff, A., and Zwirner, N.W 2003. Up-regulated expression of MICA and proinflammatory cytokines in skin biopsies from patients with seborrhoeic dermatitis. Clin. Immunol. 106:50-54.

20. Burack, W.R., Lee, K.H., Holdorf, A.D., Dustin, M.L., and Shaw, A.S. 2002. Cutting edge: quantitative imaging of raft accumulation in the immunological synapse. J. Immunol. 169:2837-2841.

21. Liu, S., Calderwood, D.A., and Ginsberg, M.H. 2000. Integrin cytoplasmic domain-binding proteins. J. Cell Sci. 113:3563-3571.

22. Monks, C.R., Freiberg, B.A., Kupfer, H., Sciaky, N., and Kupfer, A. 1998. Three-dimensional segregation of supramolecular activation clusters in T cells. Nature. 395:82-86.

23. Tadokoro, S., et al. 2003. Talin binding to integrin $\beta$ tails: a final common step in integrin activation. Science. 302:103-106.

24. Sedwick, C.E., et al. 1999. TCR, LFA-1, and CD28 play unique and complementary roles in signaling $\mathrm{T}$ cell cytoskeletal reorganization. J. Immunol. 162:1367-1375.

25. Pagano, R.E., Martin, O.C., Kang, H.C., and Haugland, R.P. 1991. A novel fluorescent ceramide analogue for studying membrane traffic in animal cells: accumulation at the Golgi apparatus results in altered spectral properties of the sphingolipid precursor. J. Cell Biol. 113:1267-1279.

26. Krummel, M.F., Sjaastad, M.D., Wulfing, C., and Davis, M.M. 2000. Differential clustering of CD4 and CD3zeta during T cell recognition. Science. 289:1349-1352.

27. de Fougerolles, A.R., and Springer, T.A. 1991. ICAM-3, a third adhesion counter-receptor for LFA-1 on resting lymphocytes. J. Exp. Med. 175:185-195.

28. Frohman, E.M., et al. 1989. Induction of ICAM-1 expression on human fetal astrocytes by interferon- $\gamma$, tumor necrosis factor- $\alpha$ and interleukin- 1 : relevance to intracerbral antigen presentation. J. Neuroimmunol. 23:117-124

29. Williams, I.R., and Kupper, T.S. 1994. Epidermal expression of intercel lular adhesion molecule 1 is not a primary inducer of cutaneous inflammation in transgenic mice. Proc. Natl. Acad. Sci. U. S. A. 91:9710-9714.

30. Coscoy, L., and Ganem, D. 2001. A viral protein that selectively downregulates ICAM-1 and B7-2 and modulates T cell costimulation. J. Clin Invest. 107:1599-1606.

31. Diefenbach, A., et al. 2002. Selective associations with signaling proteins determine stimulatory versus costimulatory activity of NKG2D. Nat Immunol. 3:1142-1149.

32. Revy, P., Sospedra, M., Barbour, B., and Trautmann, A. 2001. Functional antigen-independent synapses formed between $T$ cells and dendritic cells. Nat. Immunol. 2:925-931.

33. Marchisio, P.C., et al. 1988. Vinculin, talin, and integrins are localized at specific adhesion sites of malignant B lymphocytes. Blood. 72:830-833.

34. Teitelbaum, S.L. 2000. Bone resorption by osteoclasts. Science. 289:1504-1508.

35. Heiple, J.M., Wright, S.D., Allen, N.S., and Silverstein, S.C. 1990. Macrophages form circular zones of very close apposition to IgG-coated surfaces. Cell. Motil. Cytoskeleton. 15:260-270.

36. Loike, J.D., et al. 1992. The role of protected extracellular compartments in interactions between leukocytes, and platelets, and fibrin/fibrinogen matrices. Ann. N. Y. Acad. Sci. 667:163-172.

37. Sung, K.-L.P., Sung, L.A., Crimmins, M., Burakoff, S.J., and Chien, S. 1986. Determination of junction avidity of cytoloytic T cell and target cell. Science. 234:1405-1408

38. Chan, P.Y., et al. 1991. The influence of receptor lateral mobility on adhesion strengthening between membranes containing LFA-3 and CD2. J. Cell Biol. 115:245-255.

39. Sanderson, C.J. 1976. The mechanism of T cell mediated cytotoxicity II. Morphological studies of cell death by time-lapse microcinematography. Proc. Roy. Soc. Lond. B. 192:241-255.

40. Martz, E. 1987. LFA-1 and other accessory molecules functioning in adhesions of T and B lymphocytes. Human Immunol. 18:3-37.

41. Lee, S.J., Hori, Y., Groves, J.T., Dustin, M.L., and Chakraborty, A.K. 2002 Correlation of a dynamic model for immunological synapse formation with effector functions: two pathways to synapse formation. Trends Immunol. 23:492-499.

42. Sykulev, Y., Joo, M., Vturina, I., Tsomides, T.J., and Eisen, H.N. 1996. Evidence that a single peptide-MHC complex on a target cell can elicit a cytolytic T cell response. Immunity. 4:565-571. 